\title{
Development of a New Approach to Earthquake Prediction: Load/Unload Response Ratio (LURR) Theory \\ Xiang-Chu Yin, ${ }^{1,2}$ Yu-Cang Wang, ${ }^{1}$ Ke-Yin Peng, ${ }^{1,2}$ Yi-Long Bai, ${ }^{1}$ HAI-TAO WANG ${ }^{1}$ and XUN-FeI YIN ${ }^{1,3}$
}

\begin{abstract}
The seismogenic process is nonlinear and irreversible so that the response to loading is different from unloading. This difference reflects the damage of a loaded material. Based on this insight, a new parameter-load/unload response ratio (LURR) was proposed to measure quantitatively the proximity to rock failure and earthquake more than ten years ago. In the present paper, we review the fundamental concept of LURR, the validation of LURR with experimental and numerical simulation, the retrospective examination of LURR with new cases in different tectonic settings (California, USA, and Kanto region, Japan), the statistics of earthquake prediction in terms of LURR theory and the random distribution of LURR under Poisson's model. Finally we discuss LURR as a parameter to judge the closeness degree to SOC state of the system and the measurement of tidal triggering earthquake.

The Load/Unload Response Ratio (LURR) theory was first proposed in 1984 (YIN, 1987). Subsequently, a series of advances were made (YIN and YIN, 1991; YIN, 1993; YIN et al., 1994a,b, 1995; MARUYAMA, 1995). In this paper, the new results after 1995 are summarized (YIN et al., 1996; WANG et al., 1998a, 1999; ZHUANG and YIN, 1999).
\end{abstract}

Key words: Load/Unload Response Ratio (LURR), earthquake prediction, random distribution of LURR, intermittent SOC, tidal triggering earthquake.

\section{Introduction}

It is recognized by many scientists that the physical essence of an earthquake is precisely the failure or instability of the focal media accompanied by a rapid release of energy. Therefore the preparation process of an earthquake is exactly the deformation and damage process of the focal media.

\footnotetext{
${ }^{1}$ LNM (Laboratory for Non-linear Mechanics), Institute of Mechanics, CAS, Beijing 100036, China.

${ }^{2}$ CAP (Center for Analysis and Prediction), CSB (China Seismological Bureau), Beijing 100080,

${ }^{3}$ Water Resources and Hydropower Planning and Design General Institute, MWR (Ministry of Water Resources), Beijing 100011, China. E-mail: XYC: yinxc@btamail.net.cn; WYC: yin@Inm.imech.ac.cn; PKY: xcyin@public.bta.net.cn; BYL: baiyl@lnm.imech.ac.cn; WHT: yinxc@btamail.net.cn; YXF: xfyin@263.net
} China. 
From the microscopic viewpoint, the damage process for geo-material (rock) has incredible richness in complexity (MEAKIN, 1991; BAI et al., 1994; KrAJCINNOVIC, 1996). In any rock there must be a large number of disordered defects (cracks) with different size, shape and orientation. The damage process includes nucleation, growth, interaction, coalition and cascade of cracks. It is an irreversible, nonequilibrium and nonlinear one, which has been intensively studied for decades but a lot of fundamental questions remain still unsolved. The problem of damage and failure in solid mechanics is as difficult as the problem of turbulence in fluid mechanics, and this is the inherent difficulty of earthquake prediction.

Since the problem of damage and failure for solids is one of scientific and technological importance so that a suite of effective phenomenological methods have been developed, to which the key is the constitutive relationship or the constitutive curve of materials (JAEGER and COOK, 1976). From the macroscopic viewpoint the constitutive curve is a comprehensive description of the mechanical property of the materials. A typical constitutive curve for focal media (rock) is shown in Figure 1. For generality, in Figure 1 the ordinate denotes general load $P$ instead of stress $\sigma$ and the abscissa is the response $R$ to $P$ instead of strain $\varepsilon$. If the load acting on the material increases monotonously, the material will experience the regimes of elastic, damage and failure or destabilization. The most essential characteristic of the elastic regime is its reversibility; i.e., the positive process and the contrary process are reversible. In other words, the loading modulus and the unloading one are equal to each other. Contrary to the elastic regime, the damage one is irreversible and the loading response is different from the unloading one or the loading modulus should be different from the unloading one. This difference indicates the deterioration of materials due to damage.

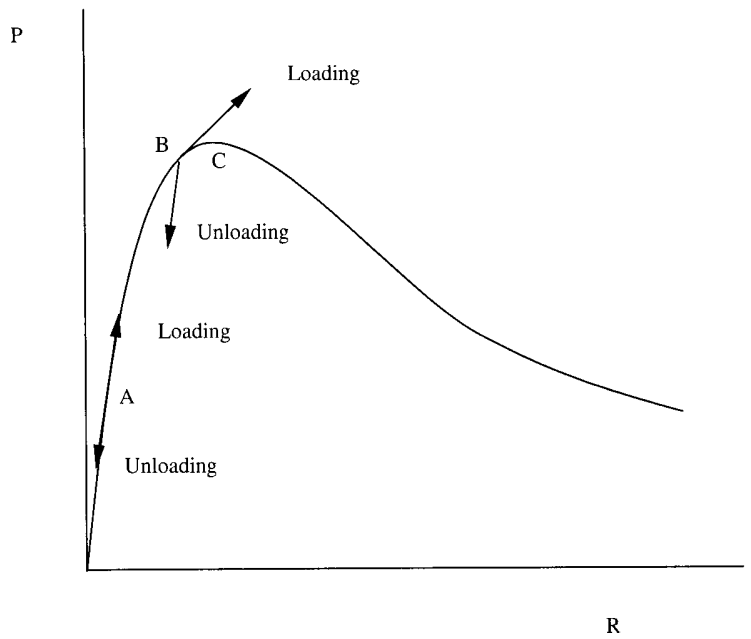

Figure 1

The constitutive curve of focal zone. 
In order to measure quantitatively the difference, two parameters are defined as follows. The first one is the response rate $X$ defined as

$$
X=\lim _{\Delta P \rightarrow 0} \frac{\Delta R}{\Delta P},
$$

where $\Delta P$ and $\Delta R$ denote the increments of load $P$ and response $R$, respectively. The second one is the Load/Unload Response Ratio (LURR) $Y$

$$
Y=\frac{X_{+}}{X_{-}},
$$

where $X_{+}$and $X_{-}$refer to response rate under loading and unloading condition, respectively.

It is clear that $Y=1$ for the elastic regime since $X_{+}=X_{-}$and $Y>1$ for the damage regime due to $X_{+}>X_{-}$. The more seriously damaged the material, the larger the $Y$ value will become. As the media approach failure the $Y$ value becomes increasingly larger so that the $Y$ value (LURR) could measure the proximity to failure and also acts as a precursor for earthquake prediction.

In continuum damage mechanics, the damage degree of material is measured by damage variable or damage parameter $D$. There are many ways to define $D$-from scalar to high order tensor (KRAJCINNOviC, 1996). A direct way is to define $D$ as the relative variation of the effective stiffness tensor. However for a simple condition, it can be simplified. According to Lemaitre's definition (LAMAitre, 1987),

$$
D=E_{0}-E / E_{0},
$$

where modulus $E_{0}$ denotes the Young's modulus for the original material and $E$ means the Young's modulus in the damaged state. It is easy to derive the relation between $D$ and $Y$ in some simplest condition (say uniaxial tension or compression). If we take the stress as $P$ and strain as $R$ in (1) and (2), then

$$
Y=E_{-} / E_{+} .
$$

Assuming the unloading modulus is equal to $E_{0}$ and the loading modulus of damaged one $E_{0}$ is $E$, then

$$
Y=E_{0} / E .
$$

Therefore we can derive a very simple relation between $Y$ and $D$ as:

$$
D=1-1 / Y .
$$

If we adopt other more complicated definitions of damage, the relation between $Y$ and $D$ is no longer as simple as (5), although there is still a functional relation between $Y$ and $D$. In other words, $Y$ is actually another damage variable. 
In fact, the reduction of modulus is due to the existence of microcracks in the material. Many scientists have studied the relation between modulus and the contained cracks. According to ODA (1983), the increase of the compliance tensor $\left(M_{i j k l}\right.$ the reciprocal of stiffness $\left.-\varepsilon_{i j}=M_{i j k l} \sigma_{k l}\right)$ could be expressed as

$$
M_{i j k l}-M_{i j k l}^{0}=(\Delta / 4)\left(\delta_{i l} F_{j k}+\delta_{j l} F_{i k}+\delta_{j k} F_{i l}+\delta_{i k} F_{j l}\right),
$$

where $M_{i j k l}^{0}$ denotes the compliance tensor of the undamaged medium, $M_{i j k j}$ denotes the compliance tensor of the damaged medium, $\delta_{i j}$ is Kronecker delta, $F_{i j}$ are the components of the fabric tensor which are defined as

$$
F_{i j}=\frac{\pi N}{V} \int_{0}^{\infty} \int_{\Omega} a^{3} D\left(n_{k}, a\right) n_{i} n_{j} d \Omega d a,
$$

where $a$ is the size of crack, $D$ is a crack distribution density function, $n$ denotes the unit normal vector and $d \Omega$ is the spherical surface element.

It is obvious that not only the calculation of the fabric tensor is very complicated, but also it is much too difficult to obtain enough data for calculating $F_{i j}$ at present. It is known that a crack or seismic fault with size $a$ corresponds to an earthquake with specified magnitude and energy (KANAMORI and ANDERSON, 1975). Therefore it would be better to define the $Y$ value directly by the seismic energy as follows:

$$
Y=\frac{\left(\sum_{i=1}^{N+} E_{i}^{m}\right)_{+}}{\left(\sum_{i=1}^{N-} E_{i}^{m}\right)_{-}}
$$

where $E$ denotes seismic energy which can be calculated according to the Gutenberg-Richter formula (KANAMORI and ANDERson, 1975; Bullen and Bolt, 1985), the sign " + " means loading and " - " means unloading, $m=0$ or $1 / 3$ or $1 / 2$ or $2 / 3$ or 1 . When $m=1, E^{m}$ is exactly the energy itself; $m=1 / 2, E^{m}$ denotes the Benioff strain; $m=1 / 3,2 / 3, E^{m}$ represents the linear scale and area scale of the focal zone, respectively; $m=0, Y$ is equal to $N^{+} / N^{-}$, and $N^{+}$and $N^{-}$denote the number of earthquake which occurred during the loading and unloading duration, respectively.

In order to predict earthquakes in terms of parameter $Y$ (LURR), a solution should be found as to load and unload the crustal blocks hundreds of kilometers in size and select proper parameters as the $R$ (response) to calculate $Y$. These problems have been elucidated in our previous papers (YIN and YIN, 1991; YIN, 1993; Yin et al., 1994a, 1995; MARUYAMA, 1995).

Here we just add two notes. The first one is the explanation of symbol $Y$. If the seismic energy is selected as $R$, then the LURR is denoted by $Y_{m}$ instead of $Y, Y_{m}$ is 


$$
Y_{m}=\frac{\left(\sum_{i=1}^{N^{+}} E_{i}^{m}\right)_{+}}{\left(\sum_{i=1}^{N^{-}} E_{i}^{m}\right)_{-}} .
$$

When the ground water lever is selected as $R$, the LURR is denoted by $Y_{w}$, for Coda Q by $Y_{c q}$, wave velocity ratio by $Y_{v-r}$, crust deformation by $Y_{d}$, geomagnetism by $Y_{g m}$, etc.

The second one is the criteria to judge loading and unloading. We adopt the Coulomb failure hypothesis to judge loading or unloading according to the sign of the increment of Coulomb failure stress which is denoted by CFS in recent literature (e.g., HARris, 1998; REASENBERG and SiMPSON, 1992).

$$
C F S=\tau_{n}+f \sigma_{n}
$$

where $f, \tau_{n}$ and $\sigma_{n}$ stand for inner frictional coefficient, shear stress and normal stress (positive in tension) respectively, $\boldsymbol{n}$ is the normal of the fault plane on which the $C F S$ reaches its maximum. This plane is parallel to the second principal stress (middle principle stress) $\sigma_{2}$ and the angle $\theta$ between the fault plane and the minimum principle stress (maximum compressive stress) $\sigma_{3}$ satisfies the following relation:

$$
\tan 2 \theta=1 / f
$$

$\triangle C F S$ is the increment of $C F S$. If the increment of Coulomb failure stress $\triangle C F S>0$, it is referred to as loading; otherwise $\triangle C F S<0$ is referred to as unloading.

It is well known that the resultant stress $\sigma_{i j}$ in the crust consists of tectonic stress $\sigma_{i j}^{T}$ and the tide induced stress $\sigma_{i j}^{t}$. Since the level of $\sigma_{i j}^{T}$ (in the order of $10^{6}-10^{8} \mathrm{~Pa}$ ) is considerably higher than the level of $\sigma_{i j}^{t}\left(10^{3}-10^{4} \mathrm{~Pa}\right)$ so the directions of the principle stress of the crust resultant stress and then the direction of $\mathbf{n}$ can be determined by the tectonic stress only. However, the change rate of tidal induced stress is much larger than the change rate of the tectonic stress (VIDALI et al., 1998) thus $\triangle C F S$ is mainly due to tidal-induced stress which could be calculated precisely. The calculation of elastic deformation of the earth can be formulated as a system of six differential equations of first order. Following and improving MolodensksyTakeuchi's work, we calculate the tide-induced stress components of any section in the crust in terms of the Runge-Kutta numerical method (Melchior, 1978; YIN and YIN, 1991). The shear and normal stress on the fault plane with normal $\mathbf{n}$ can be obtained by stress tensor transform after which the $\triangle C F S$ can be calculated easily according (10). 


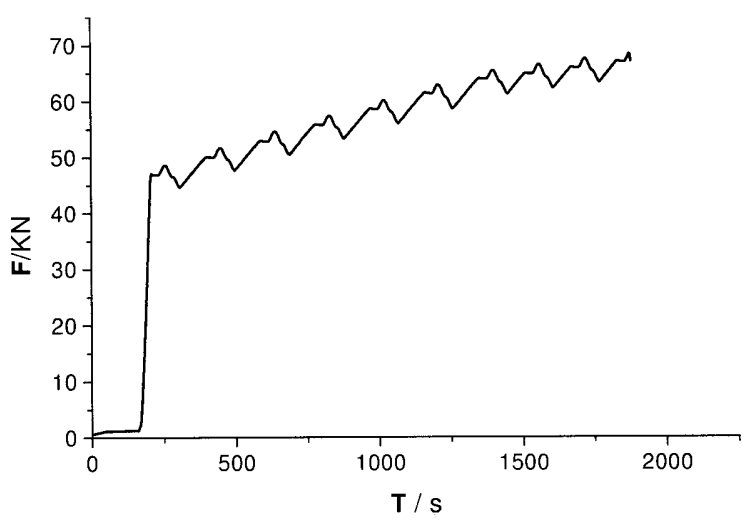

Figure 2

The $F$ - $t$ (load-time) curve.

2. Validation of LURR Theory

\section{A. Laboratory Simulation}

In order to test the validity of LURR theory, a series of rock fracture experiments have been conducted (SHI et al., 1994; WANG et al., 1998b). The specimens are rock (marble or sandstone) columns under uniaxial compression. The experiments were conducted in LNM (Laboratory for Non-linear Mechanics of Continuous Media), Institute of Mechanics, CAS and the Center Lab of USTC with test machine MTS-50. The load increases with constant rate superposing a harmonic force which simulates the tidal force (Fig. 2).

The force, displacement and the travel time are measured during the entire experiment and the variation of Young's modulus or wave velocity is taken as $R$. The experimental results are shown in Figure 3. It indicates that when the stress is at low level, the $Y$ value is always close to 1 and as soon as the specimen is damaged the $Y$ value rises. Finally the specimen fractures and $Y$ reaches a high value (considerably larger than 1). These results suggest that LURR is available as a precursor for the fracture of a brittle solid and also for an earthquake.

It is worthwhile to note that under uniaxial compression the rock material shows brittle behavior and the damage regime is very narrow so that the $Y$ value increases very steeply (refer to Fig. 5).

\section{B. Numerical Simulation}

Based on the lattice model of Mora and Place (1993), DEM (Cundall and STRACK, 1979) and MD (Molecular Dynamics approach), we developed a discrete model to simulate the damage and fracture of brittle solid (especially under compression) and in the interim measure the variation of LURR. In our model 


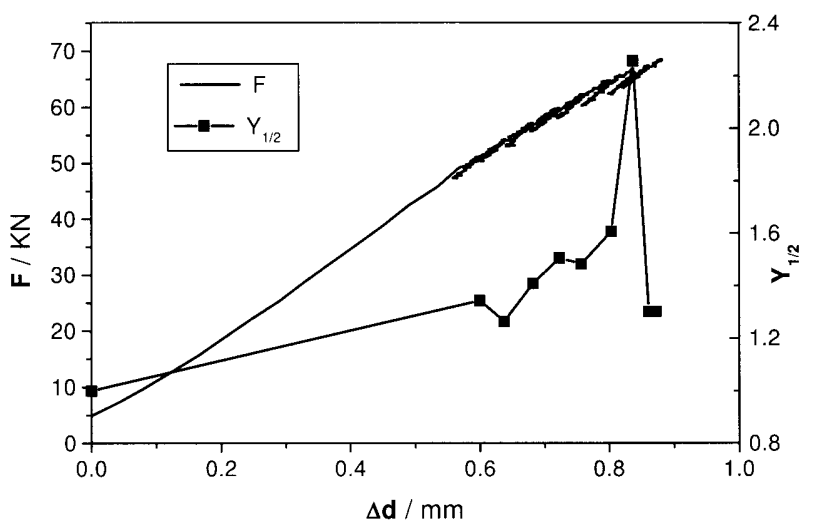

Figure 3

The variation of LURR with compressive displacement ( $Y$ - $\Delta d$ curve) during the process of damage and fracture for a marble specimen.

(WANG et al., 1999, 2000, this issue) the medium is discretized into a number of round particles which are arranged into a triangular lattice. Many particles form a mesoscopic unit with different shape and size. Any two neighboring particles interact by radial force, tangential force and bending moment. Any particle must obey the conversation laws of momentum and angular momentum. Subsequently the equations of conversation law of momentum and angular momentum can be calculated step by step with a suitable time step and the results describe the whole process underlying the damage process or earthquake process. According to equation (8), the variation of $Y$ (LURR) during the entire process can be calculated. Figure 4 is one of the simulation results. They substantiate once again that the parameter $Y$ (LURR) is actually a quantitative indicator which mirrors the

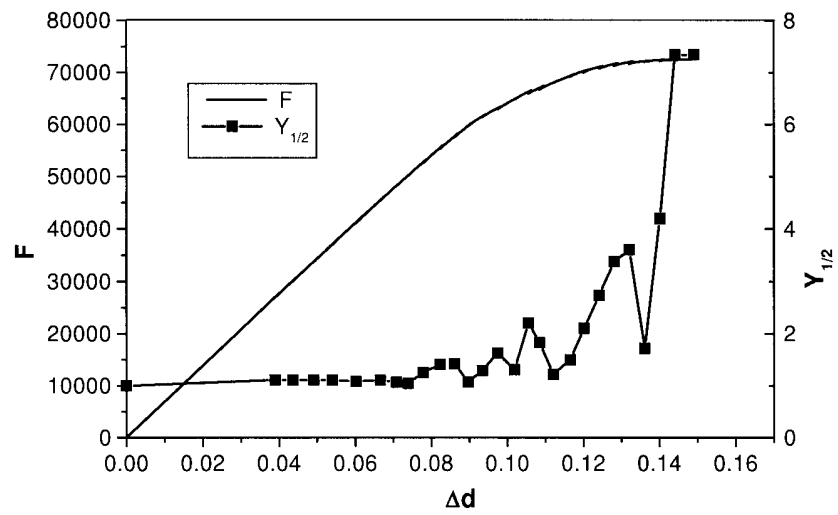

Figure 4

The numerical simulation results of variation in $Y$ during the process of damage and fracture for rock. 


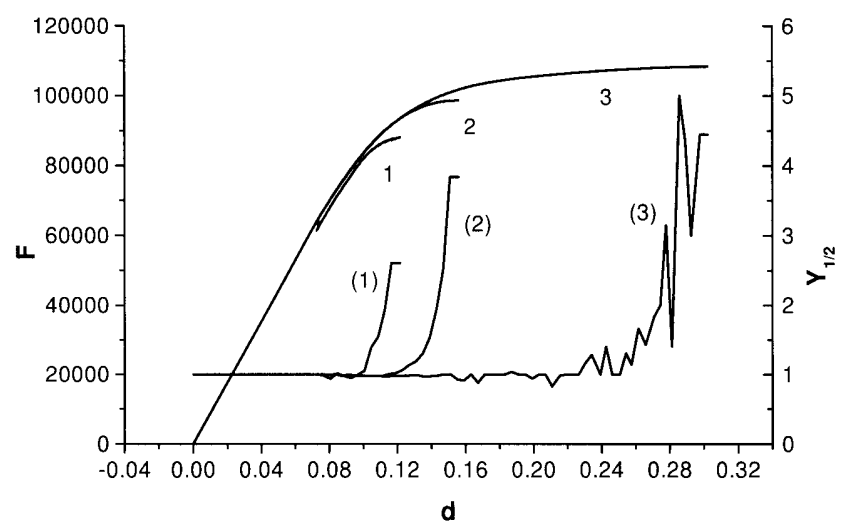

Figure 5

The influence of material heterogeneity on the variation $Y$ during the damage process.

closeness degree to instability so that it could be a precursor for earthquake prediction.

Figure 5 shows us the influence of brittleness of the medium on the behavior including the variation of $Y$. The more brittle the medium is, the more sharply the $Y$ value increases with the stress. The position of Figure 3 relates to the curve 1 or 2 in Figure 5. The influence of material heterogeneity and other factors on the damage process and the variation of $Y$ during the process (WANG et al., 1999, 2000, this issue), are also studied.

In addition, the chains network model (LIANG et al., 1996) has been adopted to simulate the damage-fracture process for rock and the evolution of LURR. The simulation results, in terms of two models, coincide beautifully.

\section{Retrospective Examination}

Although the results of laboratory experiment and numerical simulation support the validity of LURR theory very well, the most convincing way to validate the LURR theory is the retrospective examination of LURR theory with real seismic data. Hundreds of cases have been studied with the data in China (YIN et al., 1995).

In our study, usually $m=1 / 2$, spatial windows of $1^{\circ} \times 1^{\circ}$ to $2^{\circ} \times 2^{\circ}$ and temporal windows of several months to years are chosen (similarly hereinafter). Since the size of the seismogenic region scales with the size of the ensuing main shock, the linear scale $L$ of the spatial window seems to be selected according to the formula below (WANG, 1999)

$$
\log L(\mathrm{~km})=0.5 M_{s}-0.8
$$



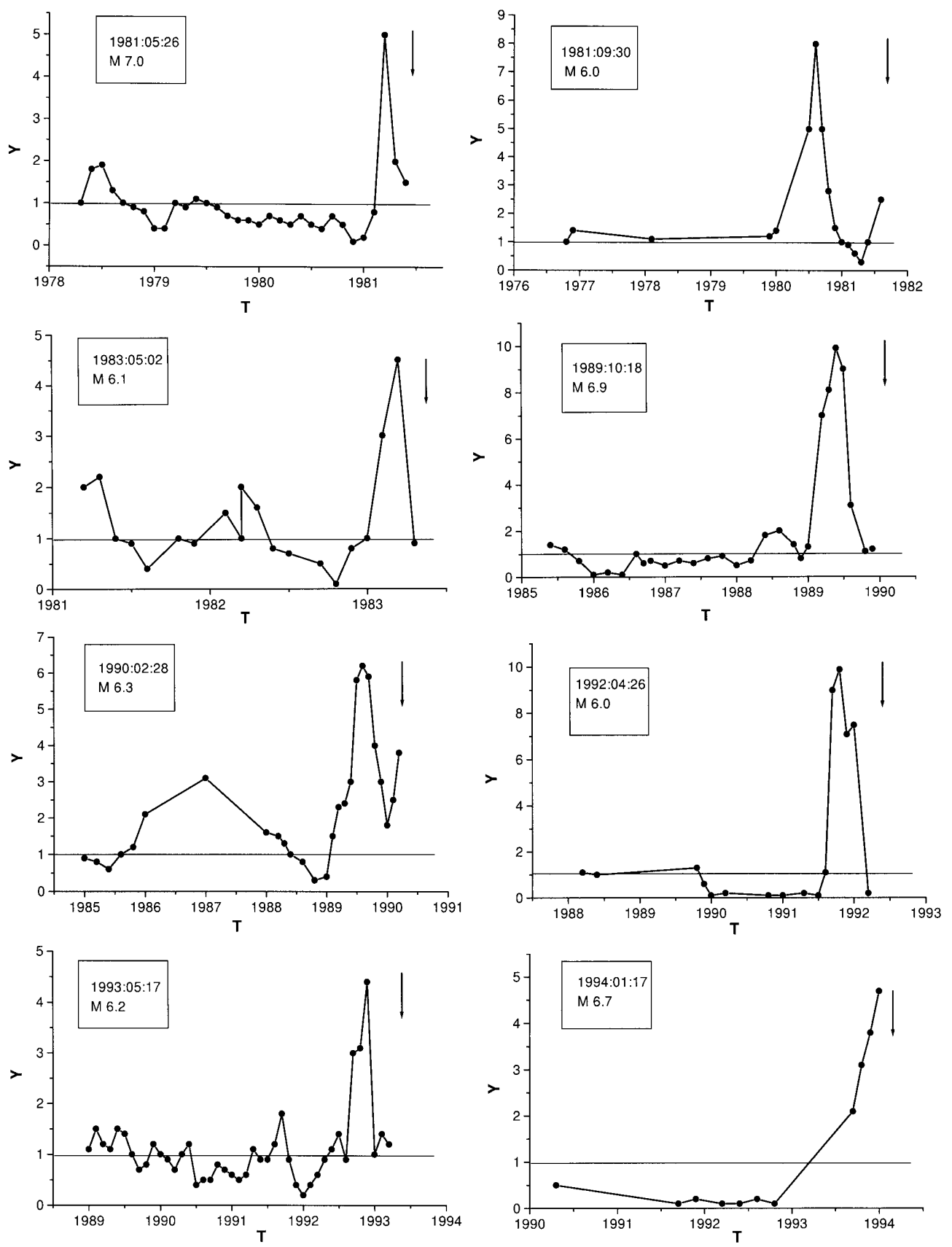

Figure 6

Variation in $Y_{1 / 2}$ with time before all strong earthquakes that occurred in southern California from 1980 to 1994 (the figure in box denotes year, month, day of occurrence and the magnitude, respectively). 

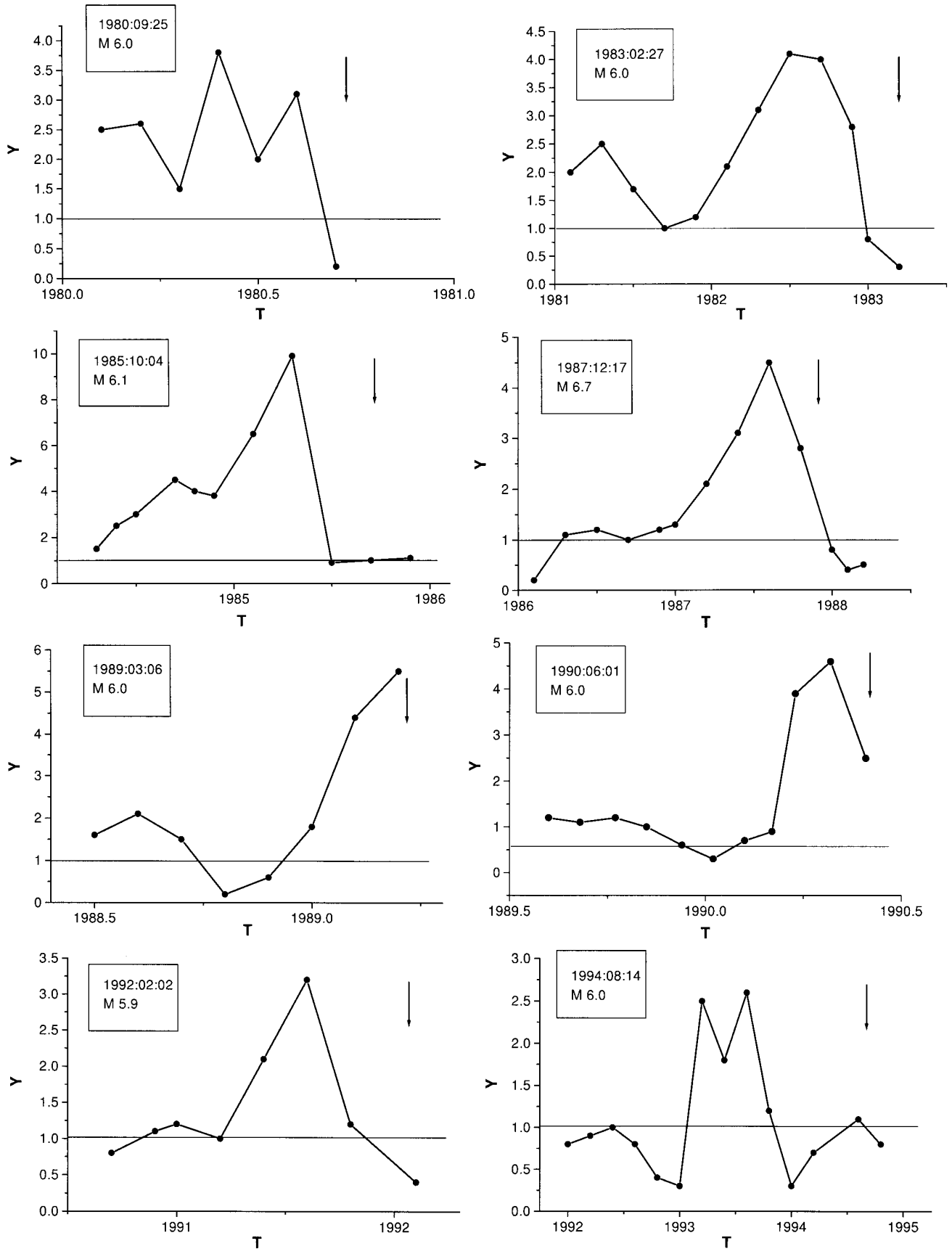

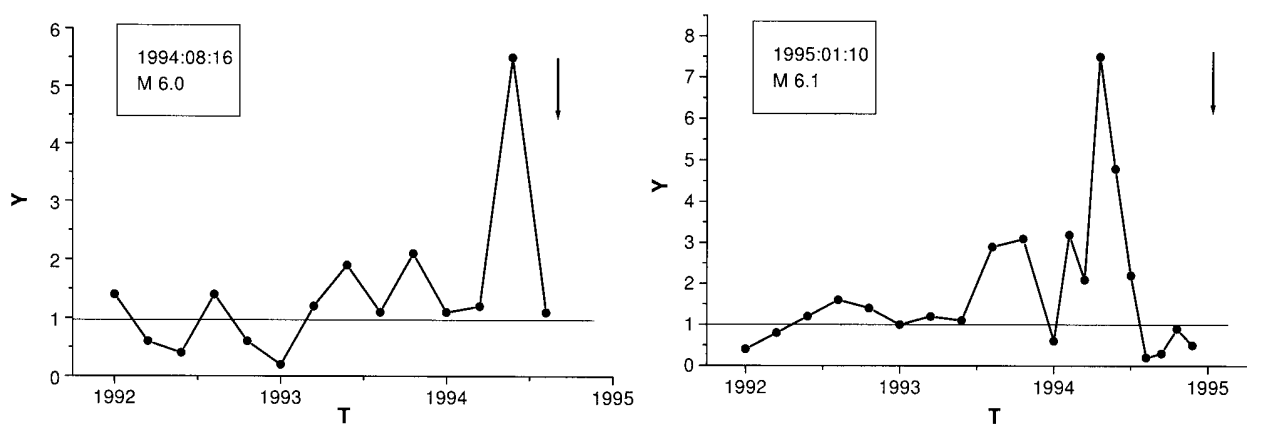

Figure 7

Variation in $Y_{1 / 2}$ with time prior to all moderate earthquakes that occurred in the Kanto region (Japan) from 1980 to 1995 (the figure in the box denotes year, month, day of occurrence and the magnitude, respectively).

where $M_{s}$ is the magnitude of the predicted main shock. The temporal window is also related to the magnitude of the predicted main shock. Another consideration is that the size of the sample, the number of earthquakes in the selected spatial and temporal windows, should be large enough. Otherwise the calculated value of LURR might be without enough confidence (refer to Fig. 10).

The results of retrospective examination indicate that the highest proportion (more than $80 \%$ ) of main shocks is preceded by a period during which the $Y$ values increase markedly and remain high values $\left(Y_{m}>1\right.$, more precisely $Y_{m}>Y_{m}^{c}$, refer to part 5 of this paper). In contrast, we selected seven "stable" regions with low seismicity (no earthquake with $M \geq 4$ occurred in the examined period) on the Chinese mainland and analyzed the variation in $Y$ for more than two decades (from 1970 to 1992). For all seven regions, the $Y$ value always fluctuates slightly around 1 during the entire duration (YIN et al., 1995).

Subsequently we have conducted further retrospective examinations for a series of strong earthquakes occurring in southern California (USA) and Kanto region (Japan). The results are shown in Figures 6 and 7, respectively. For most of the cases, the $Y$ values are significantly larger than 1 .

In addition, we have examined the variation of $Y$ in the Parkfield section of the San Andreas Fault from 1970 to 1999. It can be seen from Figure 8 that through the entire period, the $Y$ value is near unity, except for the $Y$ value in 1982-1983 which reaches to $Y_{m}^{c}$ and is a precursor for the swarm (the strongest earthquake with $M=6.3$ and many earthquakes with $M>5$ involved) which occurred in the region which is located to the northeast, and the distance between the region and Parkfield is less than 50 kilometers. The result from Figure 8 provides an explanation of why the expected earthquake around 1987 (BAKUN and LINDH, 1985; RoELOFFS and LANGBEIN, 1994) has not occurred yet. 


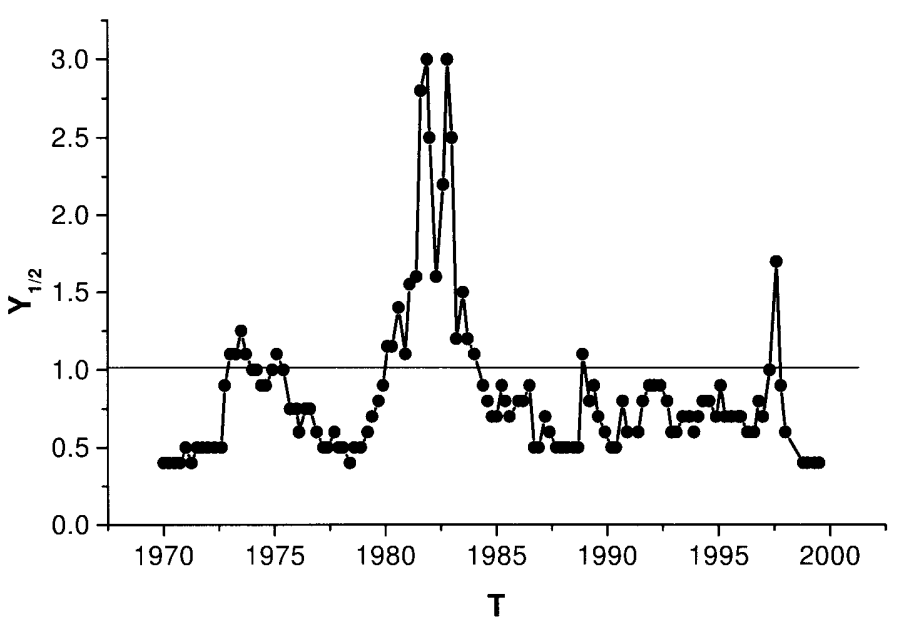

Figure 8

Variation in $Y_{1 / 2}$ in Parkfield region from 1970 to present.

It is well known that the tectonic regimes of southern California, Kanto and the Chinese mainland are quite different. The San Andreas Fault is a typical transform fault, Japan is located in a complex tectonic setting - interaction of four plates (Pacific, North American, Eurasian and Philippine Sea Plates) and most of the earthquakes occurring on the Chinese mainland are intraplate ones. The entire results of retrospective examination in different tectonic settings suggest that the $Y$ value indeed indicates the proximity to instability of a crust block for a specific region, and the LURR theory could pave the way to earthquake prediction.

\section{Tentative Practice of Earthquake Prediction}

To date, all earthquake predictions in terms of LURR theory performed by X. C. Yin and his group (not including the predictions by other people outside our group) with formal documentation, are listed in Table 1. The predicted cases are not enough for statistical testing, but Table 1 indicates that we have indeed conducted successfully some earthquake predictions (intermediate term prediction - the predicted time scale ranges from 1 month to about 1 year), using LURR with a relatively high accuracy rate.

As an example, it is worth describing the prediction of the Kanto earthquake $\left(1996.09 .11, M_{s} 6.6,35.5^{\circ} \mathrm{N}, 140.9^{\circ} \mathrm{E}\right)$. In early 1996, a scientist from JMA (Japanese Meteorological Agency) asked the first author of this paper to assist them in predicting the seismic tendency for some regions in Japan (Wakayama, Kanto, etc.) and offered us the data. After calculation and analysis of the variation of LURR $-Y(t)$ for these regions, we predicted that "a strong earthquake with a 
Table 1

Statistics of EQ prediction using LURR

\begin{tabular}{|c|c|c|}
\hline & Prediction cases & Accuracy rating \\
\hline 1 & Northridge EQ (1994.01.17, $\left.M_{s} 6.6\right)$ & \\
\hline & $\begin{array}{l}\text { Predicted on } 1993.10 .28 \text { that a "a medium EQ (which means } M_{s} 6-6.5 \text { ) would } \\
\text { occur in SA6 region within about } 1 \text { year from now on" (A letter to Dr. Eric } \\
\text { Bergman in ISOP, USGS). }\end{array}$ & $\mathrm{C}$ \\
\hline 2 & Kanto EQ $\left(1996.09 .11, M_{s} 6.6,35.5^{\circ} \mathrm{N}, 140.9^{\circ} \mathrm{E}\right)$ & \\
\hline & $\begin{array}{l}\text { Predicted in April and May of } 1996 \text { that "a strong EQ with magnitude about } \\
\text { M6 could occur in Kanto region }\left(35^{\circ}-36^{\circ} \mathrm{N} ; 139^{\circ}-141^{\circ} \mathrm{E}\right) \text { within } 1 \text { year." } \\
\text { (A Fax to Dr. Hosono Kohji, JMA and a paper published in Earthquake } \\
\text { Research and China, both Chinese and English versions) }\end{array}$ & $\mathrm{C}$ \\
\hline 3 & $\begin{array}{l}\text { Predicted on } 1994.09 .20 \text { that a felt EQ }\left(M_{L} 4-5\right) \text { would occur within } 1 \text { month } \\
\text { in BJC Region }\left(38.5^{\circ}-41^{\circ} \mathrm{N}, 115.5^{\circ}-117.5^{\circ} \mathrm{E}\right) \text { The predicted EQ did not occur } \\
\text { in the expected period. } \\
\text { Beijing EQ }\left(1994.12 .23, M_{L} 4.3 .40 .6^{\circ} \mathrm{N}, 115.6^{\circ} \mathrm{E}\right)\end{array}$ & $\mathrm{F}$ \\
\hline 4 & $\begin{array}{l}\text { Predicted on } 1994.10 .31 \text { that a felt EQ }\left(M_{L} 4-5\right) \text { would occur in } 2 \text { months in } \\
\text { BJC Region }\left(38.5^{\circ}-41^{\circ} \mathrm{N}, 115.5^{\circ}-117.5^{\circ} \mathrm{E}\right) \\
\text { Tangshan EQ }\left(1998.04 .14, M_{L} 5.0,39.7^{\circ} \mathrm{N}, 118.5^{\circ} \mathrm{E}\right)\end{array}$ & $\mathrm{C}$ \\
\hline 5 & $\begin{array}{l}\text { Predicted on } 1998.04 .06 \text { that a felt EQ about } M_{L} 5 \text { will occur within } 2 \\
\text { months in BJE Region }\left(38.5^{\circ}-41^{\circ} \mathrm{N}, 117^{\circ}-120^{\circ} \mathrm{E}\right)\end{array}$ & $\mathrm{C}$ \\
\hline 6 & $\begin{array}{l}\text { Predicted on } 1998.04 .20 \text { that an EQ with M } 5-6 \text { will occur within } 2 \text { months } \\
\text { or slightly longer in BJC region. } \\
\text { The predicted EQ has not occurred in the expected period. }\end{array}$ & $\mathrm{F}$ \\
\hline 7 & $\begin{array}{l}\text { Predicted on 1995.11.06 the seismic tendency of main China in } 1996 \text { (exactly } \\
\text { 1995.11.07-1997.01.31). }\end{array}$ & $\mathrm{C}: 3, \mathrm{~F}: 0, \mathrm{M}: 0$ \\
\hline 8 & $\begin{array}{l}\text { Predicted on 1996.11.06 the seismic tendency of mainland China in } 1997 \\
\text { (exactly 1996.11.07-1998.01.31. }\end{array}$ & $\mathrm{C}: 5, \mathrm{~F}: 2, \mathrm{M}: 0$ \\
\hline
\end{tabular}

Notes: EQ: earthquake. C: Correct prediction. F: False, the predicted EQ has not occurred. M: Missing, strong EQ occurred, but no prediction.

magnitude nearing 6 could occur in the Kanto region $\left(35^{\circ}-36^{\circ} \mathrm{N} ; 139^{\circ}-141^{\circ} \mathrm{E}\right)$ within one year." We faxed it to Dr. Hosono (JMA) and wrote a paper (both in Chinese and English) based on the above research, and submitted it to ERC (Earthquake Research in China) in April 1996 (Chinese version) and May 1996 (English version). The Chinese version of this paper was published in ERC (No. 3, 1, Sep., 1996) and its English version was published in ERC (No. 4, 1996).

According to our experience, we have made preliminary conclusions as follows: A. If the $Y$ value is low for a region, we are fully confident that no strong earthquakes will occur in the near future (say several months) in this region.

B. If the $Y$ value is high enough for a region, there are several possibilities:

1. In the majority of cases, a strong earthquake or earthquakes occur in the predicted window (time window: about 1 year, space window: about $100 \mathrm{~km}$, and its magnitude relates to the areas of high $Y$ value).

2. Sometimes the strong earthquake or earthquakes do not occur in the predicted window, but in the neighborhood of the window (not far from the window) 
3. In rare cases, no strong event occurs for a prolonged time (say after 1 year or longer).

\section{The Random Distribution of LURR}

The preparation and occurrence process of earthquakes is controlled not only by deterministic dynamical law but also is affected by stochastic or disorder factors. Therefore we must study the influence of random factors on LURR in order to judge whether the height the $Y$ value reaches can be considered as a precursor under the specified confidence (e.g., 0.95) (ZHUANG and YIN, 1999).

The Poisson model and the binomial model are used to describe the occurrence times of earthquakes. In order to save space, only the results of the Poisson model are mentioned below. We assume that earthquakes in a region obey the following basic assumption:

1. The earthquakes occur consistent with a Poisson process with a constant rate $\lambda$. The number of earthquakes occurring in the time interval $[0, T]$ has a Poisson distribution with expectation $\lambda T$, i.e.,

$$
\operatorname{Pr}\{N=n\}=\frac{(\lambda T)^{n}}{n !} e^{-\lambda t} .
$$

2. The distribution of the magnitudes obeys the Gutenberg-Richter law, i.e., an exponential distribution with the probability density function.

3. The probabilities of an earthquake falling in a loading period and unloading period are equal, both $1 / 2$.

Based on the above assumptions, a simulation algorithm for computing the distribution and the confidence bands of the LURR is outlined below:

1. For each time interval (assumed as unit time interval), simulate two random variables $P, Q$ belonging to a Poisson distribution of rate $\lambda / 2$, where $\lambda$ is the occurrence rate of earthquakes. $P, Q$ can be regarded as the number of loading earthquakes and unloading earthquakes occurring in the time interval, respectively.

2. According to the given $b$-value, simulate $P$ magnitudes for the loading earthquake and $Q$ magnitudes for the unloading earthquakes.

3. Calculate the $Y$-value.

4. Repeat steps 1 to 3 for one million times, and draw the histogram of $Y$-values, which could be regarded as the probability density function (p.d.f) of LURR. Figure 9 displays an example for $b=1, \lambda T=40$ and $m=1 / 2$.

5. Find the 0.90, 0.95 and 0.99 confidence bands from the p.d.f of LURR, respectively. Figure 10 is an example of such kind of simulated results. For example, for the condition: Occurrence rate $=50, b=1, m=1 / 2$, Confidence $95 \%, Y_{1 / 2}$ value should be equal or greater than 2.4 which denoted $Y_{m}^{c}$. 


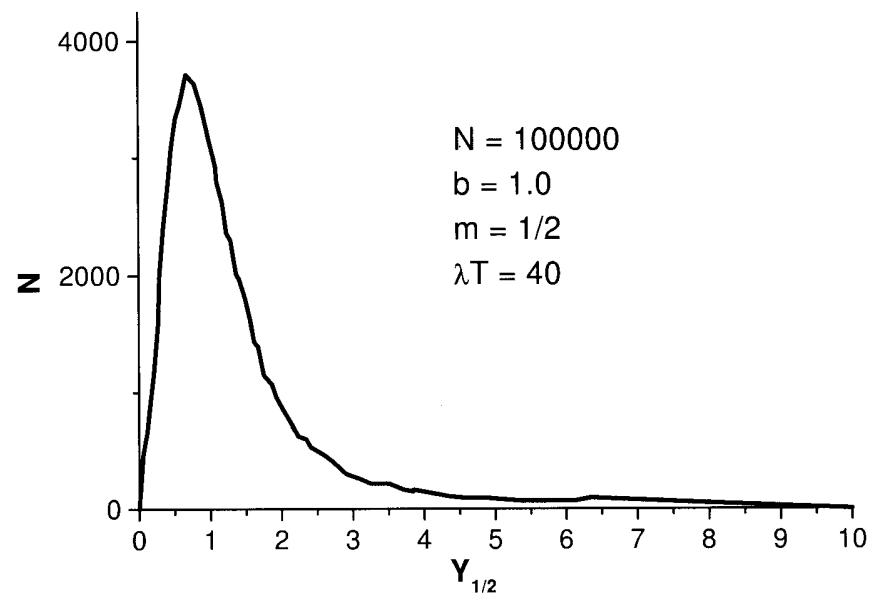

Figure 9

The random distribution of $Y_{1 / 2}$ under the specified condition.

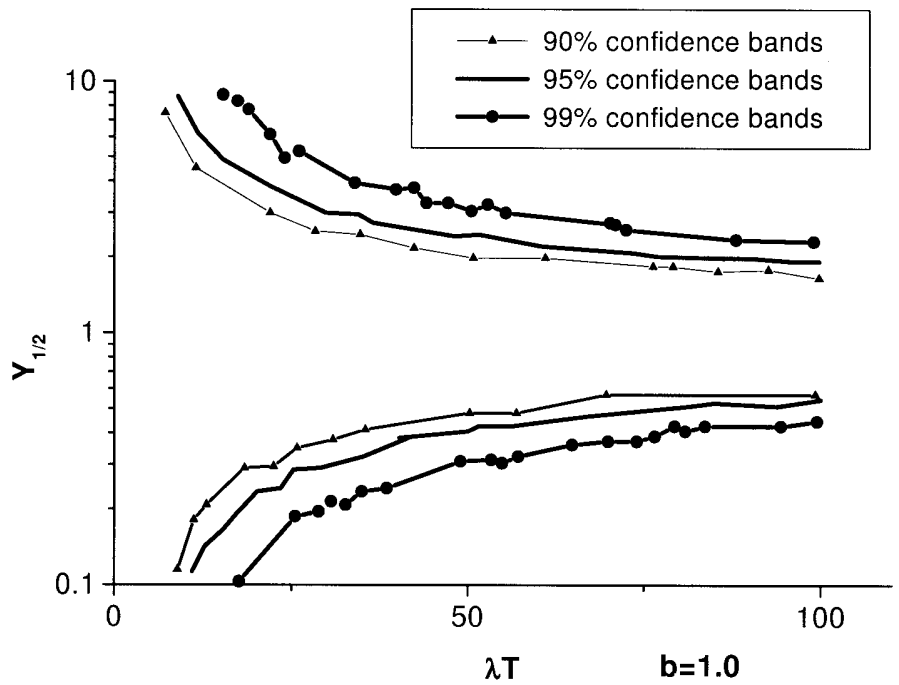

Figure 10

Variation of $Y_{1 / 2}$ with an occurrence rate under the Poisson model for specified confidence.

The results establish that the variation of LURR is controlled by the occurrence rate for the Poisson model and the parameter $S(S=b / m$, where $b$ is the constant in the Gutenberg-Richter law and $m$ is the power in (9)). The larger the occurrence rate and the $S$-value, the more stable or more concentrated around 1 the $Y$ value is. 


\section{Discussion and Conclusion}

The question of tidal triggering of earthquakes has been a controversial topic for which quite different even opposite opinions appeared in the literature (COTTON, 1922; KNOPOFF, 1964; EMTER, 1998). There is remarkable new interest in it, based on new data and viewpoints (HARRIS, 1998; VIDALI et al., 1998). In our opinion, whether the tidal stress triggers earthquakes depends on many factors such as tectonic setting, time and so on. Hence it is inappropriate to seek a stereotyped conclusion in all cases.

It is worthwhile to mention that the $Y_{m}$ value (for example, $Y_{0}=N^{+} / N^{-}$) is a suitable and convenient parameter for measuring tidal triggering of earthquakes. As shown in Figures 6 and 7, the $Y$ values are different in different periods (every $Y_{m}$ value is calculated for a specified period ranging from months to years). For the duration in which the $Y_{m}<Y_{m}^{c}$, the tidal stress does not trigger earthquakes, only in case of $Y_{m}>Y_{m}^{c}$, does tidal triggering of earthquakes take place.

The explanation of the phenomena mentioned above is simple. The tidal stress is very small: several orders smaller than the crust tectonic stress. Consequently it only could trigger earthquakes, but cannot by itself engender earthquakes in spite of the fact that the rate of stress change from earth tides exceeds that from tectonic stress accumulation (VIDALI et al., 1998). For the condition of low tectonic stress, the total stress (tectonic stress superposing the tidal stress) is still low, tidal stress is difficult to trigger any earthquake, hence the $Y_{m}$ value must be low $\left(Y_{m}<Y_{m}^{c}\right)$. On the contrary, when tectonic stress is high enough, it is very sensitive to any tiny extrinsic disturbance. Consequently the tidal stress may easily trigger earthquakes so that the $Y$ value should be high $\left(Y_{m}>Y_{m}^{c}\right)$ which generally appears prior to the occurrence of strong or large earthquakes and persists months to years and even decades (in the case of the great earthquake).

The question mentioned above is relevant to the question of whether the whole crust is in the SOC (self-organized criticality) state. We prefer the intermittent criticality hypothesis (BOWMAN et al., 1998; HUANG et al., 1998) to the hypothesis that the entire crust always stays in critical state. When a large or even great earthquake occurs just now in a region, a large portion of the cumulated energy and stress will be released so that the tectonic stress becomes low. In this case, the crust of this region is not in the critical state, its $Y$ value is low $\left(Y_{m}<Y_{m}^{c}\right)$ and tidal stress is difficult to trigger any earthquake. Thereafter the tectonic stress is accumulated gradually in this region which approaches the critical state (of course this process is not monotonic and linear). Finally it reaches the critical state in such a situation, the tectonic stress is high and the system must be sensitive to any tiny extrinsic disturbance, then the tidal stress easily triggers earthquakes so that the $Y$ value should be high $\left(Y_{m}>Y_{m}^{c}\right)$. Therefore the $Y$ value is also a parameter to represent the approaching degree to critical state. 
To sum up, the LURR theory was proposed on the basis of the constitutive relation (macroscopic phenomenological methodology). Later research brought insight to the mesoscopic viewpoint, though it still leaves many questions for future research to answer.

The LURR theory is still young therefore it has a broad room to develop. Besides seismic energy (8), many other geophysical parameters concerning the seismogenic process such as Coda $\mathrm{Q}$, ratio of velocity, level of groundwater, cubic strain tilt and strain in crust, geomagnetism parameters etc. could also be the $R$ (response) to define LURR (CHEN et al., 1994; YANG et al., 1994; CHEN and YIN, 1995; WANG, 1995; ZHEN, 1996; ZHANG, 1995). All of them have yielded interesting results and other new parameters being the new $R$ (responses), are constantly emerging.

On the other hand, LURR could be applied not only to natural earthquake prediction but also to forecasting of other geological disasters such as RIS (CHEN and YIN, 1995), MIS landslide (HUANG and QU, 1997).

As discussed above, LURR could also be an indicator measuring the proximity to the SOC state and the tectonic stress level for a specified region.

\section{Acknowledgements}

This research is supported by the Natural Science Foundation of China (Grant No. 19732060), the key project of the ninth five-year-plan of MOST (Ministry of Science and Technology) and CSB (China Seismological Bureau). We are grateful to PETER MORA for his precious suggestions. The authors are also grateful to Bernard Minster and Steven Jaumé for their enhancement of the present paper.

\section{REFERENCES}

BAI, Y. L., Lu, C. S., Ke, F. J., and XIA, M. F. (1994), Evolution Induced Catastrophe, Phys. Lett. A185, 196-201.

Bowman, D. D., Ouillen, G., Sammis, C. G., Sornette, A., and Sornette, D. (1998), An Observational Test of the Critical Earthquake Concept, J. Geophys. Res. 103, 24,359-24,372.

Bakun, W. H., and Lindh, A. G. (1985), The Parkfield, California, Earthquake Prediction Experiment, Science 229, 619-624.

Bullen, K. E., and Bolt, B. A., An Introduction to the Theory of Seismology (Cambridge University Press, Cambridge 1985).

Chen, J. M., Zhang, S. D., and Yang, L. Z. (1994), Seismic Abnormalities in LURR of Groundwater Lever, Earthquake 1, 73-78.

CHen, X. Z., and YIn, X. C. (1995), Applications of LURR Theory to the Earthquake Prediction for Reservoir-induced Earthquakes, Earthquake Research in China 11, 361-367.

Cotтon, L. A. (1922), Earthquake Frequency, with Special Reference to Tidal Stresses in the Lithosphere, Bull. Seismol. Soc. Am. 12, 47-198.

Cundall, P. A., and Strack, O. D. L. (1979), A Discrete Element Model for Granular Assemblies, Geotechnique 29, 47-65. 
Emter, D., Tidal triggering of earthquakes and volcanic events. In Tidal Phenomena (ed. Wilhelm, A.) (Elsevier, Amsterdam 1998).

HARris, R. A. (1998), Introduction to Special Section: Stress Triggers, Stress Shadows, and Implication for Seismic Hazard, J. Geophys. Res. 103, 24,347-24,358.

Huang, Y., Saleur, H., Sammis, C. G., and Sornette, D. (1998), Precursor, Aftershocks, Criticality and Self-organization Criticality, Europhys. Lett. 41, 43-48.

Huang, R. Q., and Qu, O., Scientific Analysis Principle and its Applications to Generalized System in Engineering Geology (Geological Press, Beijing 1997) (in Chinese).

JAEGer, J. C., and CooK, N. G. W., Fundamentals of Rock Mechanics (Chapman and Hall, London 1976).

Kanamori, H., and Anderson, D. L. (1975), Theoretical Basis of Some Empirical Relation in Seismology, Bull. Seismol. Soc. Am. 65, 1073-1096.

Knopoff, L. (1964), Earth Tide as a Triggering Mechanism for Earthquakes, Bull. Seismol. Soc. Am. 54, $1865-1870$.

Krajcinnovic, D., Damage Mechanics (Elsevier, Amsterdam 1996).

Lamaitre, J., Formulation and identification of damage kinetic constitutive equations. In Continuum Damage Mechanics (ed. Krajcinnovic, D.) (Springer-Verlag, Wien, New York 1987).

Liang, N., LiU, Q., Li, J., and Song, H., A Chains network model simulating meso-mechanics behavior and micro damage evolution of in situ reinforced ceramics. In Advanced in Engineering Plasticity and Its Applications (eds. Abe, T., and Tsuta, T.) (Pergamon, Amsterdam 1996) pp. 141-146.

Maruyama, T. (1995), Earthquake Prediction in China, Zisin 19 (5), 68-76.

Meakin, P. (1991), Model for Material Failure and Deformation, Science 252, 226-234.

Melchior, P., The Tide of the Planet Earth (Pergamon Press, New York 1978).

Mora, P., and Place, D. (1993), A Lattice Solid Model for the Nonlinear Dynamics of Earthquakes, Int. J. Mod. Phys. C4, 1059-1074.

ODA, M. (1983), A Method for Evaluating the Effect of Crack Geometry on the Mechanical Behavior of Cracked Rock Mass, Mech. Mater. 2, 163-171.

Reasenberg, P. A., and Simpson, R. W. (1992), Response of Regional Seismicity to the Static Stress Change Produced by the Loma Preita Earthquake, Science 255, 1687-1690.

Roeloffs, E. A., and Langbein, J. (1994), The Earthquake Prediction Experiment at Parkfield, California, Rev. Geophys. 32, 315-336.

Shi, X., Xu, H., Wan, Y., Lu, Z., and Chen, X. (1994), The Rock Fracture under Simulated Tide Force-Laboratory Study on the Loading and Unloading Response Ratio (LURR) Theory, Acta Geophys. Sin. 37, 631-636.

Vidali, J. E., Agnew, D. C., Johnston, M. J. S., and Oppenheimer, D. H. (1998), Absence of Earthquake Correlation with Earth Tides: An Indication of High Preseismic Fault Stress Rate, J. Geophys. Res. 103, 24,567-24,572.

WANG, T. W. (1995), The Application of Load/Upload Response Ratio in Earthquake Prediction by Magnetic Method, Observation and Research of Seismic Geomagnetism 16, $26-29$ (in Chinese).

WANG, H. T. (1999), The Synthetic Application of LURR Theory to Earthquake Prediction, Doctoral thesis of Institute of Geophysics, Chinese Seismological Bureau.

Wang, H. T., Peng, K. Y., Zhang, Y. X., Wang, Y. C., and Yin, X. C. (1998a), Characters of Variation of LURR During the Earthquake Sequence of Xinjiang, Chinese Sci. Bull. 43, 1752-1755.

WANG, Y. C., YIN, X. C., and WANG, H. T. (1998b), The Simulation of Rock Experiment on Load/Upload Response for Earthquake Prediction, Earthquake Research in China 14 (2), 126-130.

WANG, Y. C., YIN, X. C., and WANG, H. T. (1999), Numerical Simulation on Load/Upload Response Ratio (LURR) Theory, ACTA Geophys. Sin. (or Chinese Journal of Geophysics) 43, 511-522.

Wang, Y. C., Yin, X. C., Ke, F. J., XIA, M. F., and Peng, K. Y., (2000), Numerical Simulation of Rock Failure and Earthquake Process on Mesoscopic Scale, Pure appl. geophys. 157, 1905-1928.

YAnG, L. Z., He, S. H., and XI, Q. W. (1994), Study on the Variation of the Property of Rock Elasticity by Load/Unload Response Ratio of Tidal Volume Strain, Earthquake Research in China 10, 90-94.

YIN, X. C. (1987), A New Approach to Earthquake Prediction, Earthquake Research in China 3, 1-7 (in Chinese with English abstract).

YIN, X. C. (1993), A New Approach to Earthquake Prediction, Russia's Nature 1, 21-27 (in Russian). 
YIN, X. C., and YIN, C. (1991), The Precursor of Instability for Nonlinear System and Its Application to Earthquake Prediction, Science in China 34, 977-986.

YIN, X. C., YIN, C., and Chen, X. Z. (1994a), The precursor of instability for nonlinear system and its application to earthquake prediction-The load-unload response ratio theory. In Nonlinear Dynamics and Predictability of Geophysical Phenomena (eds. Newman, W. I., Gabrelov, A., and Turcotte, D. L.), Geophysical Monograph, 83, IUGG Volume 18, 55-60, 1994.

Yin, X. C., Chen, X. Z., Song, Z. P., and Yin, C. (1994b), The Load-unload Response Ratio Theory and its Application to Earthquake Prediction, J. Earthq. Predict. Res. 3, 325-333.

Yin, X. C., Chen, X. Z., Song, Z. P., and Yin, C. (1995), A New Approach to Earthquake Prediction: The Load/Unload Response Ratio (LURR) Theory, Pure appl. geophys. 145, 701-715.

YIN, X. C., Song, Z. P., and WAng, Y. C. (1996), The Temporal Variation of LURR in Kanto and Other Regions in Japan and Its Application to Earthquake Prediction, Earthquake Research in China 10, $381-385$.

Zhang, J. H. (1995), The Analysis of the Geomagnetic Abnormalities in Load/Unload Response Ratio Method, Observation and Research of Seismic Geomagnetism 16, 61-63 (in Chinese).

Zhen, X. P. (1996), Earthquake and Load/Unload Response Ratio of Terrestrial Magnetic Field to Solar Wind, Observation and Research of Seismic Geomagnetism 17, 49-53 (in Chinese).

Zhuang, J. C., and Yin, X. C. (1999), Random Distribution of the Load/Unload Response Ratio (LURR) Under Assumptions of Poisson Model, Earthquake Research in China 15, 128-138.

(Received August 5, 1999, revised February 26, 2000, accepted April 28, 2000) 